\title{
Study of Cherenkov Radiation from Thin Silica Aerogel
}

\section{Ken-ichi Nanbu*, Shigeru Kashiwagi, Fujio Hinode, Toshiya Muto, Ikuro Nagasawa, Ken Takahashi, Ken Kanomata, Hidetoshi Saito, Taro Abe, Yuki Saito and Hiroyuki Hama}

\section{Research Center for Electron Photon Science (ELPH), Tohoku University, Japan}

\begin{abstract}
A beam diagnostics system employing Cherenkov radiation from a thin silica aerogel has been studied at Research Center for Electron Photon Science (ELPH), Tohoku University. We are developing a Linear Focal Cherenkov ring Camera (LFC-Camera) to observe longitudinal phase space distribution of the beam extracted from a low energy RF-gun. A thin silica aerogel plate with water-free hydro-phobic treatment is being used as Cherenkov radiator. We performed the beam test of the thin silica aerogel using a $50 \mathrm{MeV}$ electron beam from t-ACTS (test accelerator as coherent terahertz source), and also we have examined performance of the thin silica aerogel for Cherenkov radiator.
\end{abstract}

\section{Keywords}

Cherenkov radiation, Beam diagnostics system, Thin silica aerogel

\section{Introduction}

Cherenkov radiation is widely used for particle detectors in nuclear physics and particle physics. We have aware that it must be a powerful tool for beam diagnostics in accelerator technology. The t-ACTS has been developed for generation of intense coherent $\mathrm{THz}$ radiation using ultra short electron beam at ELPH, Tohoku University $[1,2]$. To generate the coherent $\mathrm{THz}$ light, the electron bunch length is required to be shortened to a few hundred femtosecond or less. The t-ACTS employs velocity bunching scheme in an accelerating structure for bunch compression $[3,4]$. In the scheme, the longitudinal phase space distribution at the entrance of the accelerating structure dominates the final bunch length. The LFC-Camera will be used to diagnose the proper electron beam prior to the injection into the accelerating structure [5-7]. Cherenkov radiation from thin radiator made of silica aerogel is going to be applied for beam diagnostics of energy spread, transverse beam profile and bunch length. Preliminary results of the beam test of thin silica aerogel radiator are described in this article.

\section{LFC-Camera}

\section{Principle and concept}

The LFC-Camera employs velocity dependence of opening angle of Cherenkov light produced by electron beam to observe its energy distribution. Since the emitted time of Cherenkov light contains time information of electrons if the radiator medium is thin enough, so we can deduce the longitudinal phase space distribution by measuring both time and energy spectra simultaneously using a high resolution.

\section{Test of Thin Silica Aerogel Radiator}

\section{Thin silica aerogel radiator}

The Cherenkov radiator, which is one of the most important components in the LFC-camera system, is required to have high-transparency, low refractive index and sufficient mechanical strength. The thickness of radiator is preferred thinner, because the energy loss was diminished when passing through the radiator medium. The refractive index is also important parameter to identify the energy of the electrons. A derivative of Cheren-

*Corresponding author: Ken ichi Nanbu, Research Center for Electron Photon Science (ELPH), Tohoku University, 1-2-1 Mikamine, Taihaku-ku, Sendai 982-0826, Japan, E-mail: nanbu@Ins.tohoku.ac.jp

Received: April 29, 2017: Accepted: May 27, 2017: Published: May 29, 2017

Copyright: (c) 2017 Nanbu K, et al. This is an open-access article distributed under the terms of the Creative Commons Attribution License, which permits unrestricted use, distribution, and reproduction in any medium, provided the original author and source are credited. 


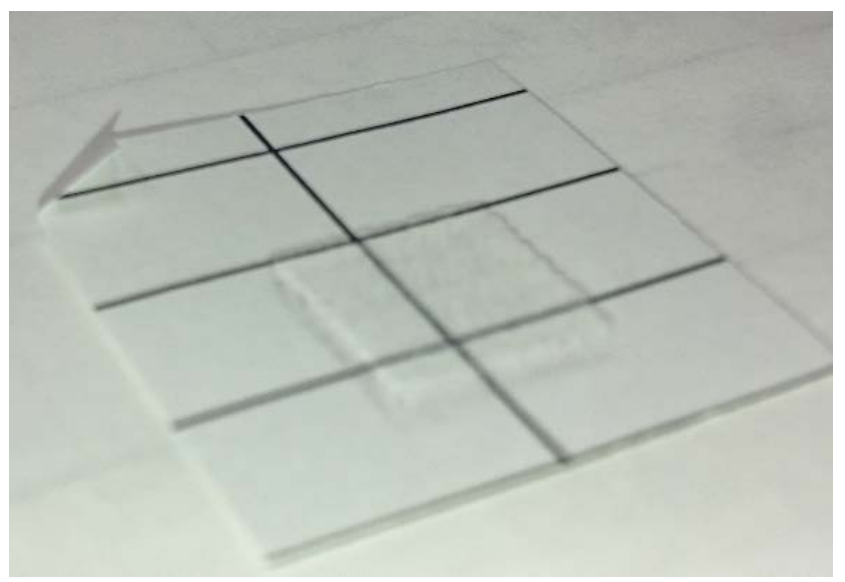

Figure 1: Photograph of thin hydrophobic silica aerogel. The dimension of this aerogel is $10 \mathrm{~mm} \times 10 \mathrm{~mm} \times 1 \mathrm{~mm}$.

kov angle respect to energy $d \theta_{c} / d E$ is preferred to be large value for precise measurements. Meanwhile, small refractive index material is not expected be mechanically strong, and the number of generated photon is smaller than one with the large refractive index. Silica aerogel (synthetic ultra light material) has some suitable characteristics. Those are not only high transparency with low refractive index but also easy processing for small thin plate. One of the candidates of low refractive index material is cryogenic liquids or compressed gases, but it was not easy to install in vacuum. Consequently, we have chosen the thin hydrophobic silica aerogel with ultra-low refractive index $(\mathrm{n}=1.05)$ developed at Chiba University $[8,9]$. Figure 1 shows a thin silica aerogel sample. Assuming no frequency dependence in both refractive index and the permeability of the Cherenkov radiator medium within narrow band, the number of emitted Cherenkov photons $N$ between wavelengths $\lambda_{1}$ and $\lambda_{2}$ is given as

$$
N=2 \pi \alpha z\left(\frac{1}{\lambda_{1}}-\frac{1}{\lambda_{2}}\right) \sin ^{2} \theta_{c},
$$

Where $z$ is radiator thickness, $\alpha$ is the fine structure constant and $\theta_{c}$ is Cherenkov angle. For example, a silica aerogel with refractive index of 1.05 and thickness of 1.0 $\mathrm{mm}$ is used as the radiator, estimated number of Cherenkov photons generates by a $20 \mathrm{pC}$ electron bunch with $\mathrm{E}$ $=2.2 \mathrm{MeV}$ at $1 \%$ bandwidth of wavelength $555 \mathrm{~nm}$ is approximately 5 Million. Overall efficiency of optical transport of the LFC-camera system is expected to be $\sim 5 \%$ of the total Cherenkov photons. Since minimum detectable photon number of the streak camera (HAMAMATSU FESCA-200) is $\sim 1000$ photons at the visible wavelength region, the number of photons is sufficient.

\section{Refractive index measurement of thin silica aerogel}

Refractive index of the thin silica aerogel is also important factor in a momentum calibration for the LFC-Camera. If refractive index of radiator changes by

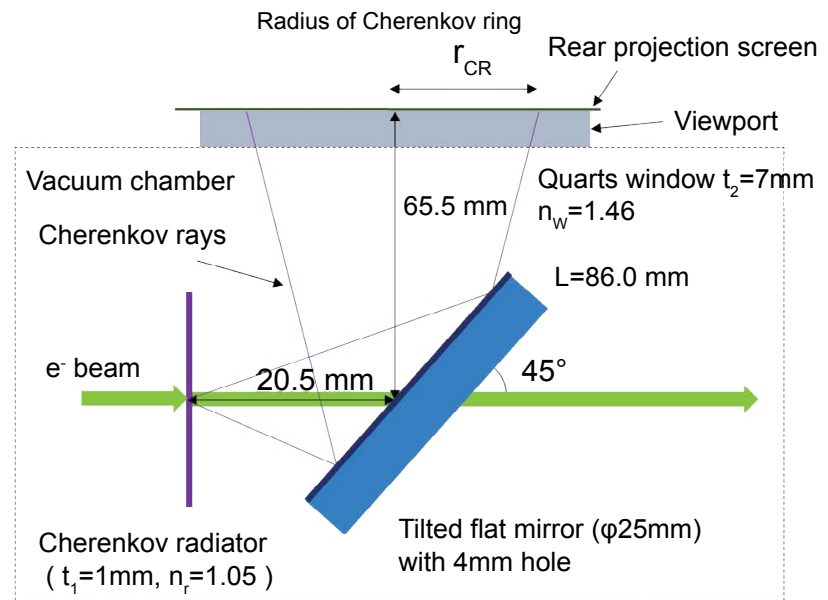

Figure 2: Schematic view of the beam irradiation test system for Cherenkov radiator. Cherenkov radiator and profile monitor are attached on the identical frame to move along the same axis. The tilted flat mirror with a $4 \mathrm{~mm}$ hole along the beam axis is installed behind Cherenkov radiator to reflect the Cherenkov light. Those components can be moved in the vacuum chamber by linear actuator with a stepper motor. The vacuum chamber is evacuated by an ion pump together a large conductance duct. The distance between surface of radiator and screen is $86.0 \mathrm{~mm}$.

only $0.1 \%$, deduced electron momentum would be shifted to $\sim 50 \mathrm{keV}$ from original one. In general, refractive index of large silica aerogel blocks for the particle counter is measured by the so-called laser fraunhofer method [9]. However, thin silica aerogel is too small to employ the laser Fraunhofer method, so that we performed measurement of the thin silica aerogel refractive index by detecting an optical path difference using a Mach-Zehnder interferometer with a $632 \mathrm{~nm}$ laser diode [10].

\section{Beam test of thin silica aerogel}

For precise longitudinal phase space measurement, ring image of the Cherenkov light is required to be not distorted. Moreover, we have to install the thin silica aerogel in ultra-high vacuum to measure the beam at the downstream of the RF-gun. To investigate the performance of the thin silica aerogel for the Cherenkov radiator, we carried out irradiation tests in the beam diagnostics section of the t-ACTS [11]. Figure 2 shows an experimental setup. Cherenkov ring profile on a screen was observed by a gated Charge-Coupled Device (CCD) camera, BASLER acA1300-gc. In the beam test, the silica aerogel radiator was irradiated by the $50 \mathrm{MeV}$ electron beam with peak current of $8 \mathrm{~mA}$ with 1 pps pulse repetition. The electron beam focused onto the radiator was measured to be $\sigma_{x}=0.23 \mathrm{~mm}$ and $\sigma_{y}=0.35 \mathrm{~mm}$. The refractive index of silica aerogel is approximately expressed by a formula

$$
n=1+k \rho \text {, }
$$

Where $k$ is a constant number and $\rho$ is its density. In 
case of the refractive index of silica aerogel is 1.05; its density is $0.2 \mathrm{~g} / \mathrm{cm}^{3}$ from Equation (2). A Continuous

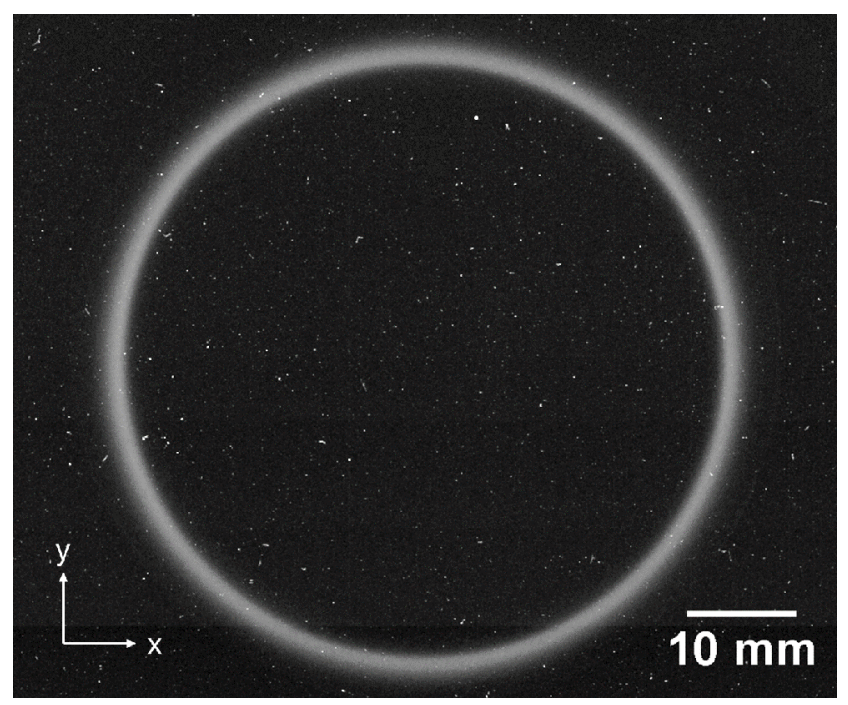

Figure 3: Spatial image of Cherenkov ring on the screen.
Slowing Down Approximation (CSDA) range of the silica aerogel is estimated to be about $1000 \mathrm{~mm}$ for $50 \mathrm{MeV}$ electron beam by its density. The energy loss of electron beam is $68 \mathrm{keV} / \mathrm{cm}$ at $50 \mathrm{MeV}$ [12]. The thickness of the Cherenkov radiator used in the experiment is only $1 \mathrm{~mm}$, which is $0.1 \%$ equivalent in the CSDA range and $0.14 \%$ equivalent in the total energy loss, respectively. Therefore, the effect of beam loss for Cherenkov angle when passing through the Cherenkov radiator is assumed to be negligible small. Figure 3 shows an observed Cherenkov ring on a screen. Measured profile data are analyzed using an ellipse function to evaluate the property of Cherenkov ring profile, and then we derived the radius of $28.4 \mathrm{~mm}$ for both the horizontal and the vertical directions. Assuming no alignment error of components, the radius of the Cherenkov ring on screen is given by

$r_{C R}=t_{1} \tan \theta_{c}+\left(L-t_{1}-t_{2}\right) \cdot \frac{n_{r} \sin \theta_{c}}{\sqrt{1-\left(n_{r} \sin \theta_{c}\right)^{2}}}+t_{2} \cdot \frac{n_{r} \sin \theta_{c}}{n_{w} \sqrt{1-\left(\frac{n_{r}}{n_{w}} \sin \theta_{c}\right)^{2}}}$
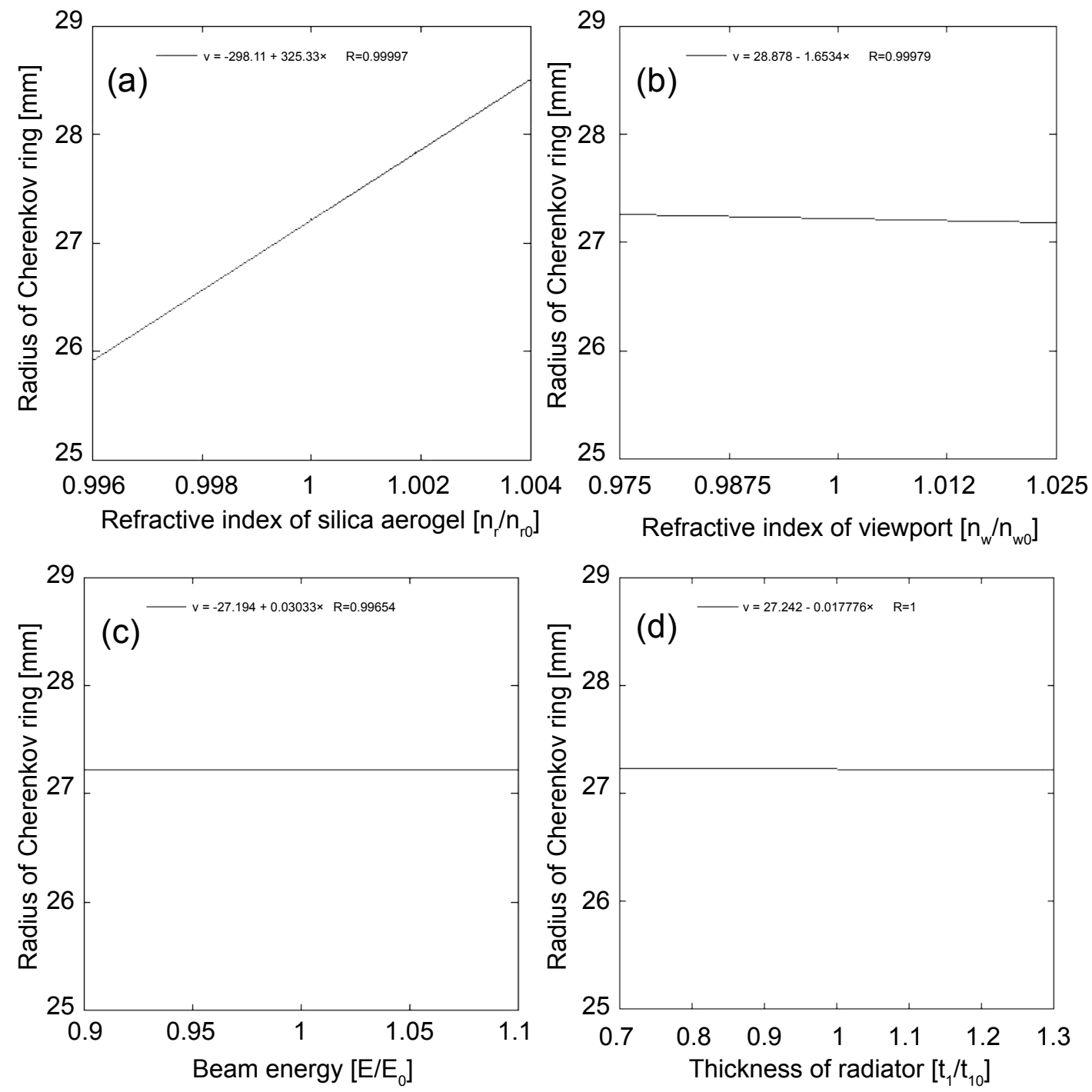

Figure 4: Solid lines denote variation of the radius of Cherenkov ring on the screen for different parameters where $n_{r 0}=1.05$, $\mathrm{n}_{\mathrm{w} 0}=1.46, \mathrm{E}_{0}=50[\mathrm{MeV}]$ and $\mathrm{t}_{10}=1[\mathrm{~mm}]$ 
Where $L$ is total pass length, $t_{1}$ is thickness of radiator, $\mathrm{t}_{2}$ is thickness of window, $\mathrm{n}_{\mathrm{r}}$ and $\mathrm{n}_{\mathrm{w}}$ are refractive indexes of the radiator and a viewport, respectively. The estimated radius of Cherenkov ring on the screen in this experimental configuration is $28.2 \mathrm{~mm}$ from Equation (3). The measured radius of the Cherenkov ring was almost consistent with the expected one. Figure 4 shows possible effects of parameter errors in the experimental configuration. Figure 4 indicates that the radius of the Cherenkov ring depends strongly on the refractive index of Cherenkov radiator, another parameters has little influence on the radius of the Cherenkov ring.

Much outgas from the Cherenkov radiator was observed during the beam irradiation. We have not found significant change on the radiator surface after irradiation. However, there might be a change in a physical property of silica aerogel. A change in refractive index of silica aerogel has a huge impact on the radius of Cherenkov ring. By monitoring of the ring radius, the variation of the refractive index of radiator can be detected that is significant to derive the absolute value of electron momentum.

\section{Conclusion and Prospect}

We have been developing the beam diagnostics system at t-ACTS using Cherenkov radiation emitted from the thin silica aerogel. We examined the performance of the thin silica aerogel Cherenkov radiator. The observed Cherenkov ring was not distorted and its radius was almost consistent with the expected one. Since Cherenkov light is two orders of magnitude brighter than an Optical Transition Radiation (OTR), we can detect using the usual gated CCD camera without special device such as an Image Intensifier (IIT). Although we noticed that the vacuum pressure in the radiator chamber was increased by the outgas from the silica aerogel during irradiation, the ultra-high vacuum environment near the RF-gun will be not much affected. It can be concluded that Cherenkov radiation emitted from thin silica-aerogel is very powerful tool for the beam diagnostics system. We continue to develop the LFC-camera, and will try to measure the bunch length as a first step. In addition, it is also planned to develop a real-time profile monitor system employing the thin silica aerogel for high energy beams.

\section{Acknowledgment}

We greatly appreciate Dr. M. Tabata of Chiba University for providing the thin hydrophobic silica aerogel samples. This work was supported by JSPS KAKENHI Grant Numbers JP15K13394 and JP15K17486.

\section{References}

1. H Hama, H Tanaka, N Kumagai, M Kawai, F Hinode, et al. (2006) Conceptual design of an isochronous ring to generate coherent terahertz synchrotron radiation. New Journal of Physics 8: 292.

2. Hiroyuki Hama, Masayuki Kawai, Shigeru Kashiwagi, Fujio Hinode, Fusashi Miyahara, et al. (2011) Test-accelerators as coherent terahertz source program (t-ACTS) at Tohoku University. Energy Procedia 9: 391.

3. L Serafini, M Ferrario (2001) Velocity Bunching in Photo-Injectors. AIP Conf Proc 581: 87.

4. S Kashiwagi, S Nagasawa, F Hinode, T Muto, $H$ Saito, et al. (2016) Proof-of-Principle Experiment of Velocity Bunching for Ultra-short Electron Pulse Production. Energy Procedia 89: 346-352.

5. H Hama, K Nanbu, M Kawai, S Kashiwagi, F Hinode, et al. (2010) Cherenkov ring to observe longitudinal phase space of a low energy electron beam extracted from RF gun. Proc of BIW10, 79-83.

6. K Nanbu, S Kashiwagi, M Kawai, F Hinode, X Li, et al. (2011) Study of reflective optics for Ifc-camera. Proc of FEL2011, 576-578.

7. A Lueangaramwong (2014) "Development of Linear Focal Cherenkov-ring Camera for Direct Observation of Longitudinal Phase Space of Non-relativistic Electron Beam". Master's thesis, Tohoku University.

8. Makoto Tabata, Ichiro Adachi, Yoshikazu Ishii, Hideyuki Kawai, Takayuki Sumiyoshi, et al. (2010) Development of transparent silica aerogel over a wide range of densities. Nucl Instr and Meth A 623: 339.

9. Makoto Tabata, Ichiro Adachi, Hideyuki Kawai, Takayuki Sumiyoshi, Hiroshi Yokogawa (2012) Hydrophobic silica aerogel production at KEK. Nucl Instr and Meth A 668: 64.

10. K Nanbu, K Kashiwagi, H Hinode, Y Shibasaki, T Muto, et al. (2014) Linear focal cherenkov-ring camera for single shot observation of longitudinal phase space distribution for non-relativistic electron beam. Proc of IBIC2014, 385-388.

11. K Nanbu, S Kashiwagi, F Hinode, T Muto, I Nagasawa, et al. (2016) Development status of linear focal cherenkov ring camera. Proc of IPAC2016, 152-154.

12. http://physics.nist.gov/PhysRefData/Star/Text/intro.html 